\title{
SOME TRIPLE EQUATIONS INVOLVING INVERSE FINITE MELLIN TRANSFORMS
}

\author{
by JOHN TWEED
}

(Received 4th January 1973)

\section{Introduction}

In this paper we investigate some triple equations involving the inverse of the finite Mellin transform $M_{R}$ which is defined by the equation

$$
M_{R}[f(x) ; s]=\int_{0}^{R}\left[x^{s-1}+R^{2 s} x^{-s-1}\right] f(x) d x .
$$

This transformation is one of four which were first introduced by $D$. Naylor in his paper (1) and some of its properties have been listed by the author in paper (2), where its relationship to the Mellin transform is discussed in detail.

\section{The triple integral equations}

In this section we shall consider the equations

$$
\begin{aligned}
M_{R}^{-1}\left[s^{-1} A(s) ; x\right] & =0, & & 0<x<a \\
M_{R}^{-1}[A(s) \cot (\pi s) ; x] & =f(x), & & a<x<b \\
M_{R}^{-1}\left[s^{-1} A(s) ; x\right] & =0, & & b<x<R
\end{aligned}
$$

where $|\operatorname{Re}(s)|<1$.

Let

$$
M_{R}^{-1}\left[s^{-1} A(s) ; x\right]=\int_{a}^{b} H(x-t) p(t) d t
$$

then clearly the first and third of the equations (2.1) will be satisfied automatically provided

$$
\int_{a}^{b} p(t) d t=0
$$

From (1.1) and (2.2) we see that

$$
s^{-1} A(s)=\int_{a}^{b}\left(x^{s-1}+R^{2 s} x^{-s-1}\right) d x \int_{a}^{x} p(t) d t
$$

and hence that $A(\mathrm{~s})$ is given by the formula

$$
A(s)=\int_{a}^{b} p(t)\left[R^{2 s} t^{-s}-t^{s}\right] d t
$$


If we now substitute from (2.4) into the second of the equations (2.1) and make use of the result

$$
M_{R}\left[\frac{R^{2}}{R^{2}-x t}-\frac{t}{t-x} ; s\right]=\pi\left(R^{2 s} t^{-s}-t^{5}\right) \cot (\pi s), \quad|\operatorname{Re}(s)|<1
$$

we find that it too will be satisfied if

$$
\frac{1}{\pi} \int_{a}^{b} p(t)\left[\frac{R^{2}}{R^{2}-x t}-\frac{t}{t-x}\right] d t=f(x), \quad a<x<b .
$$

If we let $\tau=t / R, \alpha=a / R, \beta=b / R$ and then put $\rho=x / R$ and $R / x$ in turn, we find that (2.5) implies that

$$
\frac{R}{\pi} \int_{\alpha}^{\beta} p(R \tau)\left[\frac{\tau}{1-\rho \tau}-\frac{1}{\tau-\rho}\right] d \tau= \begin{cases}\rho^{-1} f(R \rho), & \alpha<\rho<\beta \\ \rho^{-1} f\left(R \rho^{-1}\right), & \alpha^{-1}<\rho<\beta^{-1}\end{cases}
$$

and hence that

$$
\frac{1}{\pi} \int_{\alpha}^{\alpha^{-1}} \frac{h(\tau)}{\tau-\rho} d \tau=g(\rho), \quad \rho \in(\alpha, \beta) \cup\left(\beta^{-1}, \alpha^{-1}\right)
$$

where the bar on the integral sign is used to indicate that the range of integration excludes the interval $\left(\beta, \beta^{-1}\right)$ and where we have written

and

$$
h(\tau)= \begin{cases}-R p(R \tau), & \alpha<\tau<\beta \\ R \tau^{-2} p\left(R \tau^{-1}\right), & \beta^{-1}<\tau<\alpha^{-1}\end{cases}
$$

$$
g(\rho)= \begin{cases}\rho^{-1} f(R \rho), & \alpha<\rho<\beta \\ \rho^{-1} f\left(R \rho^{-1}\right), & \beta^{-1}<\rho<\alpha^{-1} .\end{cases}
$$

The equation (2.7) has been investigated by a number of authors (3), (4) and (5). In particular Lewin (5) has shown that its solution may be written in the form

$$
h(\tau)=\frac{\operatorname{sgn}\left(2 \beta \tau-1-\beta^{2}\right)}{\Delta(\alpha, \beta, \tau)}\left\{C_{0}+C_{1} \tau-\frac{1}{\pi} f_{\alpha}^{\alpha^{-1}} \frac{\Delta(\alpha, \beta, r) g(r) d r}{\operatorname{sgn}\left(2 \beta r-1-\beta^{2}\right)(r-\tau)}\right\}
$$

where

$$
\Delta(\alpha, \beta, \tau)=\left[(\tau-\alpha)\left(\alpha^{-1}-\tau\right)(\beta-\tau)\left(\beta^{-1}-\tau\right)\right]^{\frac{1}{2}}
$$

and $C_{0}$ and $C_{1}$ are arbitrary constants. In order to determine these constants we make use of condition (2.3) which clearly implies that

$$
\int_{\alpha}^{\alpha^{-1}} h(\tau) d \tau=0 \text { and } \int_{\alpha}^{\beta} h(\tau) d \tau=0 .
$$

On applying the first of these conditions and making use of the results (5), we have

$$
\frac{1}{\pi} \int_{\alpha}^{\alpha^{-1}} \frac{t^{n} \operatorname{sgn}\left(2 \beta t-1-\beta^{2}\right)}{\Delta(\alpha, \beta, t)} d t= \begin{cases}1, & n=1 \\ 0, & n=0\end{cases}
$$


and

$$
\frac{1}{\pi} \int_{\alpha}^{\alpha^{-1}} \frac{\operatorname{sgn}\left(2 \beta t-1-\beta^{2}\right) d t}{\Delta(\alpha, \beta, t)(t-r)}=0, \quad r \in(\alpha, \beta) \cup\left(\beta^{-1}, \alpha^{-1}\right)
$$

we find that $C_{1}=0$. Similarly, on applying the second condition, we discover that

$$
C_{0} \int_{\alpha}^{\beta} \frac{d \tau}{\Delta(\alpha, \beta, \tau)}=\frac{1}{\pi} \int_{\alpha}^{\beta} \frac{d \tau}{\Delta(\alpha, \beta, \tau)} \int_{\alpha}^{\alpha^{-1}} \frac{\Delta(\alpha, \beta, r) g(r) d r}{\operatorname{sgn}\left(2 \beta r-1-\beta^{2}\right)(r-\tau)} .
$$

From these results and (2.10) it now follows that $h(\tau)$ is given by the equation

$$
\begin{aligned}
& h(\tau)=\frac{\operatorname{sgn}\left(2 \beta \tau-1-\beta^{2}\right)(1-\alpha \beta)}{\Delta(\alpha, \beta, \tau) 2 \pi(\alpha \beta)^{\frac{1}{2}} F} \\
& \quad \times \int_{\alpha}^{\alpha^{-1}} \frac{\Delta(\alpha, \beta, r) g(r) d r}{\operatorname{sgn}\left(2 \beta r-1-\beta^{2}\right)(r-\tau)} \int_{\alpha}^{\beta}\left(\frac{\xi-\tau}{r-\xi}\right) \frac{d \xi}{\Delta(\alpha, \beta, \xi)},
\end{aligned}
$$

where $F$ is the complete elliptic integral of the first kind $F[\pi / 2,(\beta-\alpha) /(1-\alpha \beta)]$. If we now change back to the original variables we find that $p(t)$ is given by the formula

$$
\begin{aligned}
p(t)=\frac{\left(R^{2}-a b\right)}{2 \pi F \Delta(t)}\left\{\int_{a}^{b} \frac{R^{2} \Delta(x) f(x) d x}{\left(R^{2}-x t\right) x} \int_{a}^{b}\right. & \frac{(z-t) d z}{\left(R^{2}-z x\right) \Delta(z)} \\
& \left.-\int_{a}^{b} \frac{\Delta(x) f(x) d x}{(x-t) x} \int_{a}^{b} \frac{(z-t) d z}{(x-z) \Delta(z)}\right\}
\end{aligned}
$$

with

$$
\Delta(t)=\left[(t-a)\left(R^{2}-a t\right)(b-t)\left(R^{2}-b t\right)\right]^{\frac{t}{t}},
$$

which together with (2.2) yields $A(s)$.

\section{REFERENCES}

(1) D. NAYLoR, On a Mellin type integral transform, J. Math. Mechs. 12 (1963), 265-274.

(2) J. TWEed, Glasgow Math. J. (to appear).

(3) F. G. Tricomi, Z. Angew. Math. Phys. 2 (1951), 402.

(4) I. Palócz, The integral equation approach to currents and fields in plane parallel transmission lines, J. Math. Mechs. 15 (1966), 541-559.

(5) L. LEwIN, The solution of singular integral equations over a multiple interval and applications to multiple diaphragms in rectangular waveguide, SIAM J. Appl. Math. 16 (1968), 417-438.

\section{UNIVERSITY OF GLASGOW}

